\title{
MALAT-I is Associated with the Doxorubicin Resistance in U-2OS Osteosarcoma Cells
}

\author{
Chang Liu ${ }^{1,2, *}$ \\ Xuesong $\operatorname{Han}^{1}, *$ \\ Bo $\mathrm{Li}^{2, *}$ \\ Shaobin Huang' \\ Zhong Zhou' \\ Zhiwei Wang ${ }^{2}$ \\ Wanming Wang'
}

'Department of Orthopedics, The 900th Hospital of Joint Logistic Support Force, Fuzhou, Fujian Province, 350025, People's Republic of China; ${ }^{2}$ Department of Orthopedics, Changhai Hospital Affiliated to the Naval Medical University, Shanghai, 200433, People's Republic of China

*These authors contributed equally to this work
Correspondence: Wanming Wang Department of Orthopedics, The 900th Hospital of Joint Logistic Support Force,

I56 North Xi-er Huan Road, Gulou

District, Fuzhou, Fujian Province, 350025,

People's Republic of China

Tel +86-13705007328

Email wangwm93@I26.com

Zhiwei Wang

Department of Orthopedics, Changhai Hospital Affiliated to the Naval Medical University, 168 Changhai Road, Yangpu

District, Shanghai, 200433, People's

Republic of China

Tel +86-13918766347

Email wang123@smmu.edu.cn
Purpose: Our study aimed to investigate the relationship between MALAT-1 (metastasisassociated lung adenocarcinoma transcript 1) expression and the chemotherapy drug resistance in osteosarcoma.

Methods: The U-2OS osteosarcoma cell line was selected for the experiment. The cells were treated with methotrexate, doxorubicin, cisplatin, and ifosfamide, respectively. RT-PCR was applied to detect the MALAT-1 expression in cells. The doxorubicin-resistant cell line was constructed. The cells were divided into doxorubicin-sensitivity group (DS/shCtrl), doxorubicin-resistance group (DR/shCtrl) and shMALAT1-doxorubicin-resistance group (DR/shMALAT1). The colony formation assay and 5-ethynyl-2'-deoxyuridine (EdU) assay were used to detect cell proliferation. PI staining was used to detect the cell cycle. Transwell assay and wound healing assay were used to observe the migration and invasion ability. Annexin V-FITC assay was used to detect cell apoptosis. Western blot was used to detect the protein expression and potential mechanism. The impacts of MALAT-1 expression were verified in vivo.

Results: The MALAT-1 was upregulated in the doxorubicin-resistant U-2OS osteosarcoma cells. Downregulating MALAT-1 in the doxorubicin-resistant cells inhibited the proliferation, migration, and invasiveness, increased the ratio of cells in the G0/G1 phase, promoted apoptosis. In the doxorubicin-resistant U-2OS cells, the extracellular regulated protein kinases (ERK) phosphorylation was declined, which could be reversed by downregulating MALAT-1. In vivo assay indicated that the growth of doxorubicin-resistant solid osteosarcoma could be suppressed by downregulating MALAT-1.

Conclusion: Our study provides evidence that doxorubicin may upregulate MALAT-1 in osteosarcoma. Downregulating MALAT-1 in the doxorubicin resistance U-2OS cells could reverse the resistance and may improve chemotherapeutic efficiency. Some conclusions in previous literature may be one-sided.

Keywords: osteosarcoma, doxorubicin, long noncoding RNA, MALAT-1, chemotherapy resistance, extracellular regulated protein kinases

\section{Introduction}

Osteosarcoma is the most common primary malignant tumor of bone in children and young adults. ${ }^{1}$ It is highly aggressive and prone to local recurrence and early lung metastasis, resulting in high mortality and disability. The current treatment for osteosarcoma is surgery combined with perioperative chemotherapy. Neoadjuvant chemotherapy is a leap in the osteosarcoma treatment, which has increased the 5-year survival rate to $60-75 \%$ in patients without distant metastases at diagnosis ${ }^{2}$ and enabled more than $80 \%$ limb-salvage rate for extremity osteosarcoma patients. ${ }^{2,3}$ However, in osteosarcoma patients with recurrence or metastasis, it remains 
disappointed that the 5-year event-free survival is approximately $20 \%$, which has not improved in more than 25 years despite aggressive surgical resection and intensive systemic chemotherapy. ${ }^{1,4}$ Chemotherapy resistance is another issue, which reduces the chemotherapeutic efficiency and leads to a poor prognosis.

Molecular targeted therapy and gene immunotherapy may be the hot spots for osteosarcoma therapy. Recently, the role of long noncoding RNAs (lncRNAs) in tumorigenesis and tumor progression has been widely concerned. Despite a lack of protein-coding capacity, lncRNAs regulate gene expression at multiple levels and plenty of them play notable roles in regulating cancer biology. ${ }^{5,6}$ LncRNA MALAT-1 (Metastasis-associated Lung Adenocarcinoma Transcript 1) is originally identified as an overexpressed transcript in early-stage metastasizing non-small cell lung cancer. $^{7}$ Previous studies revealed that MALAT-1 is overexpressed in osteosarcoma specimens and is closely related to the prognosis. ${ }^{8-11}$ Downregulating MALAT-1 expression can significantly delay the growth, reduce the invasion and migration, inhibit metastasis in osteosarcoma cells. ${ }^{9-13}$ Several studies also found that MALAT-1 is associated with chemotherapy resistance in some malignant tumors, such as cervical cancer, ${ }^{14}$ hepatocellular carcinoma, ${ }^{15}$ large B-cell lymphoma, ${ }^{16}$ and colorectal cancer. ${ }^{17}$

However, the correlations between MALAT-1 and chemotherapy resistance in osteosarcoma are still unknown. Explore the inner relationship may be helpful to improve the chemotherapeutic efficiency. The U-2OS cell line was selected in our study, as it is widely used in osteosarcoma researches and has been confirmed to have relatively higher MALAT-1 expression among several osteosarcoma cell lines. ${ }^{11,18}$ In our study, we initially explored the effects of four commonly used chemotherapeutic drugs on MALAT-1 expression in the U-2OS cells. Then, we further explored the correlations between MALAT-1 expression and doxorubicin resistance in vitro and in vivo. To our knowledge, this might be the first research on the correlation between MALAT-1 and osteosarcoma chemotherapy resistance, and it may reveal some one-sided conclusions in previous literature.

\section{Materials and Methods Cell Culture}

The human U-2OS osteosarcoma cell line was purchased from Immocell Biotechnology Co., Ltd (Xiamen, China). The chemotherapeutic drugs were purchased from Yunhao
Biological Technology Co., Ltd (Fuzhou, China). All the cells were maintained in a DMEM medium containing $10 \%$ Fetal Bovine Serum (Gemini-Bio, CA, USA). To explore the effects of chemotherapeutic drugs on MALAT-1 expression, the cells were seeded on a 96-well plate, treated with a progressively higher concentration of different drugs (methotrexate, doxorubicin, ifosfamide, and cisplatin) respectively, then cultured in a $37^{\circ} \mathrm{C}$ incubator with $5 \% \mathrm{CO}_{2}$ for 24 and 48 hours.

\section{Construction of Doxorubicin-Resistant Cells}

U-2OS osteosarcoma cells were collected in the logarithmic growth phase and seeded in culture flasks. The doxorubicin at $0.03 \mu \mathrm{mol} / \mathrm{L}$ was used initially to induce the drug resistance when the cells were $70 \%$ to $80 \%$ confluent. After being cultured for 24 hours, cells were cleaned twice by PBS and cultured in a complete culture medium without doxorubicin. When the cells fully recovered, the doxorubicin was added again at $0.04 \mu \mathrm{mol} / \mathrm{L}$. According to the above-mentioned method, the concentration of doxorubicin was increased by $0.01 \mu \mathrm{mol} / \mathrm{L}$ each time until the cells could grow stably for one week at a drug concentration of $2 \mu \mathrm{mol} / \mathrm{L}$.

\section{Real-Time Polymerase Chain Reaction (RT-PCR)}

RNA Isolater Total RNA Extraction Reagent (Vazyme Biotech Co., Ltd, Nanjing, China) was used to extract RNA. Subsequently, RNA was reverse transcribed into cDNA using HiScript II Q RT SuperMix for qPCR kit (Vazyme Biotech Co., Ltd, Nanjing, China) according to the manufacturer's protocol. RT-PCR was then performed to detect the expression of MALAT-1. 18s RNA gene was used as the internal reference gene. The primers used were as follows: MALAT-1: forward: 5'-GACGGAG GTTGAGATGAAGC-3' and reverse: 5'-ATTCGGG GCTCTGTAGTCCT-3'. 18s: forward: 5'- CGACGAC CCATTCGAACGTCT $-3^{\prime}$ and reverse: $5^{\prime}$ - CTCTCCGG AATCGAACCCTGA- $3^{\prime}$. The $2^{-\Delta \Delta \mathrm{Ct}}$ method was employed to analyze the results.

\section{Knockdown of MALAT-I in Drug-Resistant U-2OS Osteosarcoma Cells}

The selected drug-resistant cells were inoculated into a 6-well plate at a concentration of $2 \times 10^{5}$ cells/well the day before transfection. The MALAT-1 shRNA and 
Table I The Primer Sequence for Knockdown of MALAT-I in Drug-Resistant Cells

\begin{tabular}{|l|l|c|}
\hline Name & & Sequences \\
\hline MALATI-Targetl & Forward & 5'-GAUCCAUAAUCGGUUUCAA-3' \\
\hline MALATI-TargetI & Reverse & 5'- UUGAAACCGAUUAUGGAUC -3' \\
\hline MALATI-Target2 & Forward & 5'- UUAGCGGAAGCUGAUCUCCAA -3' \\
\hline MALATI-Target2 & Reverse & 5'- UUGGAGAUCAGCUUCCGCUAA -3' \\
\hline Negative control & Forward & 5'- UUCUCCGAACGUGUCACGUTT -3' \\
\hline Negative control & Reverse & 5'- ACGUGACAGGUUCGGAGAATT -3' \\
\hline
\end{tabular}

negative control (NC) were synthesized by Hippo Biotechnology Co., Ltd (Huzhou, China). When cells reached $40-60 \%$ confluence, the plasmid containing MALAT-1 shRNA or NC were transfected using Lipofectamine ${ }^{\mathrm{TM}} 2000$ transfection reagent (Invitrogen, CA, USA) following the protocol recommended by the manufacturer. Their sequences are shown in Table 1. Cells were collected 48 hours after transfection for further analysis.

\section{Colony Formation Assay}

The cells were inoculated into a 6 -well plate at a density of $2 \times 10^{3}$ cells per well and cultured in a $37^{\circ} \mathrm{C}$ incubator with $5 \% \mathrm{CO}_{2}$. When visible clones appeared, the cells were fixed with $4 \%$ paraformaldehyde for 15 minutes and then stained with $0.01 \%$ crystal violet for 30 minutes. The colony number was counted.

\section{5-Ethynyl-2'-Deoxyuridine (EdU) Assay}

EdU Cell Proliferation Kit (RiboBio Biotech Co., Ltd, Guangzhou, China) was employed following the manufacturer's instructions. In brief, the cells were inoculated into a 96-well plate at a density of $10 \times 10^{3}$ cells per well and incubated with Edu medium for 2 hours. Next, the cells were fixed with paraformaldehyde and incubated with $50 \mu \mathrm{L}$ glycine $(2 \mathrm{mg} / \mathrm{mL})$. After that, the cells were incubated with $100 \mu \mathrm{L}$ PBS containing $0.5 \%$ TritonX-100. Then the cells were stained with Apollo and Hoechst staining solution sequentially. The fluorescence microscope was used for observation.

\section{Cell Cycle Assay}

PI staining was used to detect the cell cycle. The cells were washed with cold D-Hanks solution $\left(4^{\circ} \mathrm{C}\right)$ and fixed with cold $75 \%$ ethanol $\left(4^{\circ} \mathrm{C}\right)$ for $12 \mathrm{~h}$. After being washed with cold D-Hanks solution again, the cells were suspended with cell staining solution $1 \mathrm{~mL}$. The formulation of the staining solution was as follows: $40 \times$ PI mother solution $(2 \mathrm{mg} / \mathrm{mL}$, Sigma, USA): $100 \times$ RNase mother solution $(10 \mathrm{mg} / \mathrm{mL}$, Fermentas, MD, USA): $1 \times$ D-Hanks $=25: 10: 1000$. After incubation in the dark for $30 \mathrm{~min}$ at $4^{\circ} \mathrm{C}$, the percentages of the stained cells in each phase were measured by flow cytometry (Millipore, MA, USA).

\section{Transwell Migration Assay and Invasion Assay}

The migration ability of cells was measured using the Transwell chamber (Corning, NY, USA). Briefly, transfected cells were added to upper chambers in FBS-free medium, and medium with $20 \%$ FBS as chemo-attractant was added in lower chambers. After incubation at $37^{\circ} \mathrm{C}$ with $5 \% \mathrm{CO} 2$ for $24 \mathrm{~h}$, the Transwell chamber was taken out and the cells on the upper surface were removed by cotton buds. Cells on the lower surface were fixed with $4 \%$ polymethanol for 30 minutes and stained with $0.1 \%$ crystal violet for 20 minutes.

In the invasion assay, the Transwell chamber was coated with Matrigel (pore size $8.0 \mu \mathrm{m}$, BD Bioscience, CA, USA) and the remaining procedure was similar to that of the migration assay.

\section{Wound Healing Assay}

The cells were inoculated into 6-well plates. After $24 \mathrm{~h}$ in culture, scratches perpendicular to the plates were made using a transfer liquid gun and the width of all scratches was required to be about the same. The cell fragments produced by the scratch were washed and $4 \mathrm{~mL}$ serum-free medium was added. After being cultured in a $37^{\circ} \mathrm{C}$ incubator with $5 \% \mathrm{CO}_{2}$ for 48 hours, wound healing was observed. 


\section{Cell Apoptosis Assay}

Cell apoptosis was detected using an Annexin V-FITC apoptosis assay kit (AAT Bioquest Inc., CA, USA). In brief, after being washed with cold D-Hanks solution $\left(4^{\circ} \mathrm{C}\right)$ and $1 \times$ binding buffer solution, the cells were resuspended with $200 \mathrm{uL} 1 \times$ binding buffer. Then, $2 \mathrm{uL}$ Annexin V-APC was added and the cells were incubated at room temperature in the dark for 20 minutes. Finally, cells were detected by flow cytometry (Millipore, MA, USA).

\section{Western Blot}

Cells were lysed with RIPA lysis buffer containing proteasome inhibitor on ice. Protein concentrations were quantified using a BCA protein assay kit (EpiZyme, Shanghai, China). Equal amounts of protein $(20 \mu \mathrm{g})$ were separated by $10 \%$ SDS-PAGE and transferred onto polyvinylidene fluoride (PVDF) membranes. The PVDF membranes were blocked by $5 \%$ nonfat milk and then incubated with the primary antibody (Proteintech Group, Wuhan, China) at $4^{\circ} \mathrm{C}$ overnight. After being washed with TBST, the membranes were incubated with horseradish peroxidase (HRP)conjugated secondary antibodies (Proteintech Group, Wuhan, China) at room temperature for 2 hours. The bands were visualized by ECL kit (EpiZyme, Shanghai, China) and the grayscale values were measured by Image J software (National Institutes of Health, USA).

\section{In vivo Tumorigenic Assay}

Male athymic BALB/c nude mice (6-7 weeks) were purchased from Vital River Laboratory Animal Technology Co., Ltd (Beijing, China). The mice were housed and fed under specific pathogen-free conditions. A total of $3 \times 10^{6}$ cells were implanted subcutaneously into the right legs of mice. Tumor volume was calculated using the formula: Tumor volume $=\left(\right.$ length $\times$ width $\left.^{2}\right) / 2$. On day 30, mice were killed, tumors were collected and weighed.

\section{Statistical Analysis}

Experimental values were obtained from at least three independent experiments. The statistical analysis was conducted with SPSS 19.0 (IBM Inc, IL, USA). Measurement data were presented as mean $\pm \mathrm{SD}$. Comparisons between two groups were conducted using the two-tailed Student's $T$-test. Multiple sets of data were analyzed using the One-way ANOVA and LSD-t-test. Differences were considered to be statistically significant when the P value was less than 0.05 .

\section{Results}

The Effects of Chemotherapeutic Drugs on MALAT-I Expression in U-2OS

\section{Osteosarcoma Cells}

The U-2OS cells were treated with methotrexate (Figure 1A), doxorubicin (Figure 1B), cisplatin (Figure 1C), and ifosfamide (Figure 1D) at different concentrations respectively. The MALAT-1 expression changed in some conditions. In this initial exploration, we found that the cells treated with doxorubicin at a concentration of $1.0 \mathrm{umol} / \mathrm{L}$ appeared a good trend of MALAT-1 change. Based on these results, doxorubicin was selected for the following exploration.

\section{The MALAT-I Was Upregulated in the Doxorubicin-Resistant U-2OS Osteosarcoma Cells}

To further explore the correlations between MALAT-1 expression and doxorubicin resistance in osteosarcoma, the doxorubicin-resistant U-2OS cell line was constructed. We discovered that MALAT-1 was upregulated in the doxorubicin-resistant U-2OS osteosarcoma cells when compared with the original doxorubicin-sensitive U-2OS cells (Figure 2A).

\section{Proliferation Was Suppressed in Doxorubicin-Resistant U-2OS Cells with Downregulated MALAT-I Expression}

Two targets were selected to downregulate MALAT-1 expression in the doxorubicin-resistant U-2OS osteosarcoma cells. The target- 1 demonstrated a higher efficiency and was selected for subsequent study (Figure 2B).

We initially explored the proliferative ability using clone formation assay. The number of colonies (Figure 2C and D) illustrated that the doxorubicinresistant U-2OS osteosarcoma cells had higher proliferation. Downregulating MALAT-1 suppressed the proliferation.

EdU assay (Figure 2E and F) and the cell cycle assay (Figure $2 \mathrm{G}$ and $\mathrm{H}$ ) were used for further exploration. It revealed that more cells were in the proliferative stage in the doxorubicin-resistant cells. Downregulating MALAT-1 drove the doxorubicin-resistant cells into G0/G1 phase and decreased the percentage of cells in the $\mathrm{G} 2 / \mathrm{M}$ phase. 

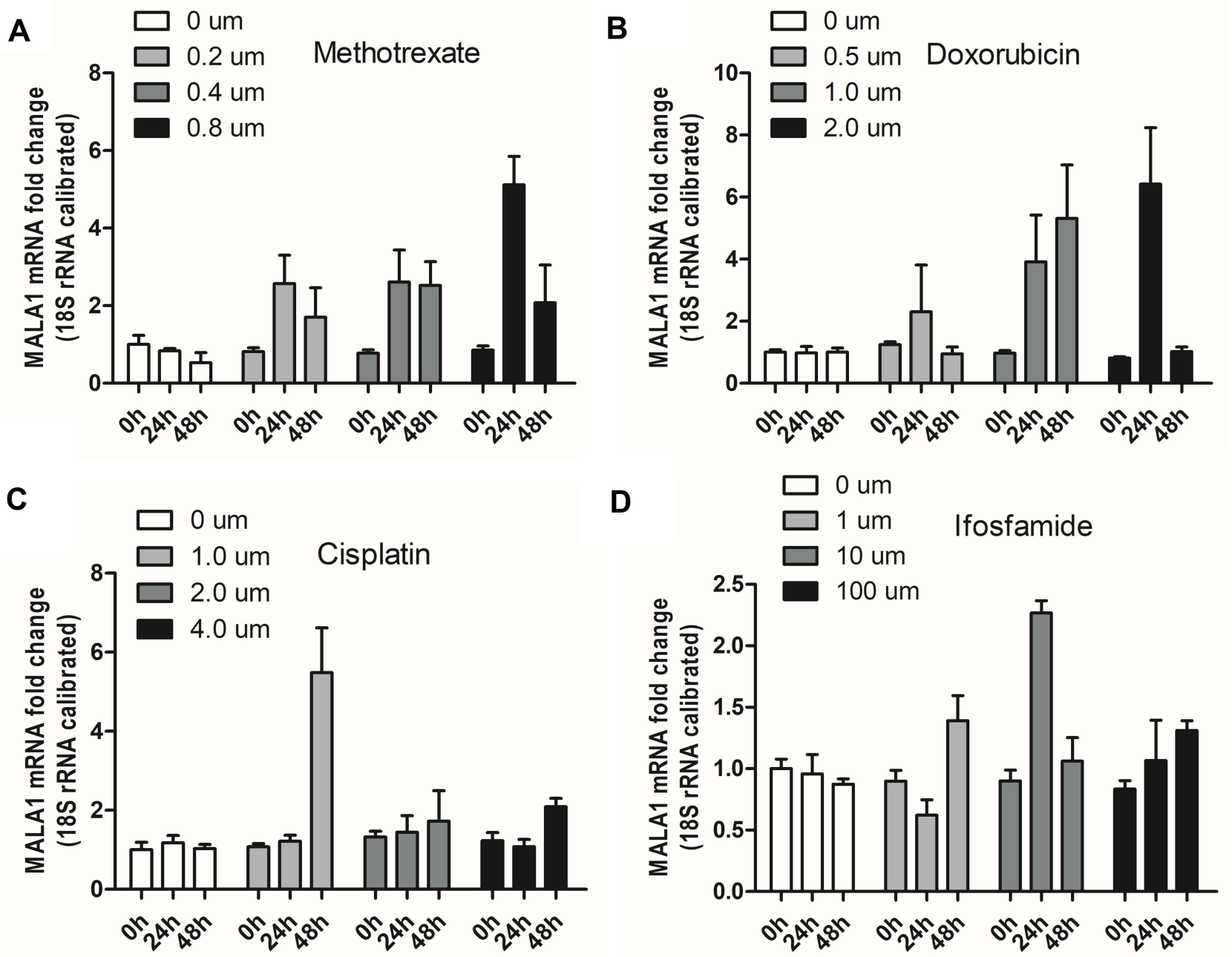

Figure I Effects of methotrexate (A), doxorubicin (B), cisplatin (C), ifosfamide (D) on MALAT-I expression in U-2OS osteosarcoma cells. The cells treated with doxorubicin demonstrated a relatively good trend of upregulated MALAT-I.

\section{Cell Migration and Invasiveness Were Inhibited in Doxorubicin-Resistant U-2OS Cells with Downregulated MALAT-I Expression}

As demonstrated in the wound healing assay (Figure 3A), doxorubicin-resistant U-2OS cells behaved with a better scratch healing ability, which was reversed by the inhibition of MALAT-1. Similarly, the migration assay unveiled that the efficient migration ability of doxorubicin-resistant U-2OS cells was weakened by downregulating MALAT-1 (Figure 3B and C).

As the invasion assay exhibited (Figure 3D and E), the doxorubicin-resistant U-2OS cells have a higher invasion ability and can be inhibited by upregulating MALAT-1.
Downregulating MALAT-I Promoted the Apoptosis and Extracellular Regulated Protein Kinases (ERK) Phosphorylation in Doxorubicin-Resistant U-2OS Cells

As demonstrated in flow cytometry (Figure 4A and B), the percentage of apoptotic cells decreased in the doxorubicinresistant U-2OS cells. Downregulating MALAT-1 expression led to more apoptosis.

By performing Western blot analysis, we observed that the doxorubicin-resistant U-2OS cells had a lower pERK/ ERK ratio. In the doxorubicin-resistant U-2OS cells, the expression of Caspase 3 and Bax was downregulated and the expression of Bcl-2 was upregulated. Inhibiting MALAT-1 promoted the phosphorylation of ERK and 
A

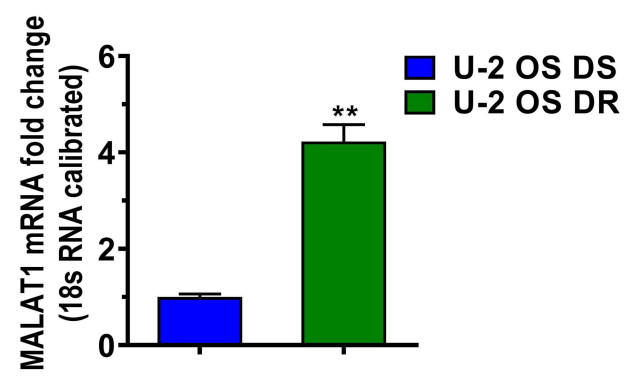

C

\section{U-2 OS DS/shCtrl}

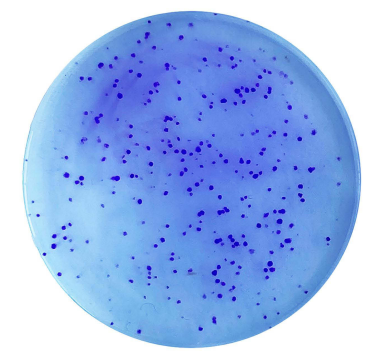

E

\section{U-2 OS DS/shCtrI}

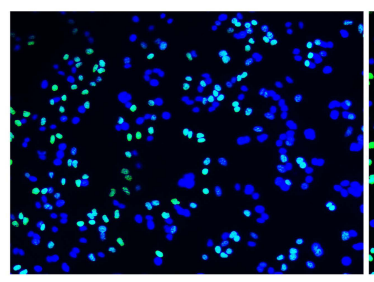

G

\section{U-2 OS DS/shCtrl}

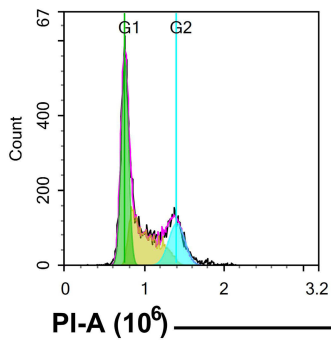

U-2 OS DR/shCtrl

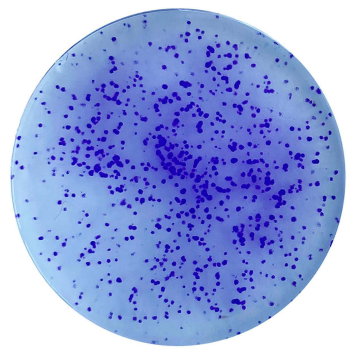

U-2 OS DR/shCtrl

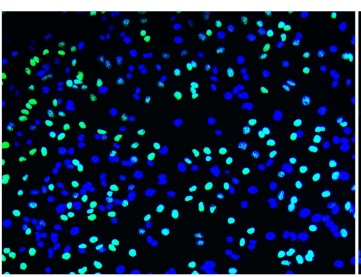

B

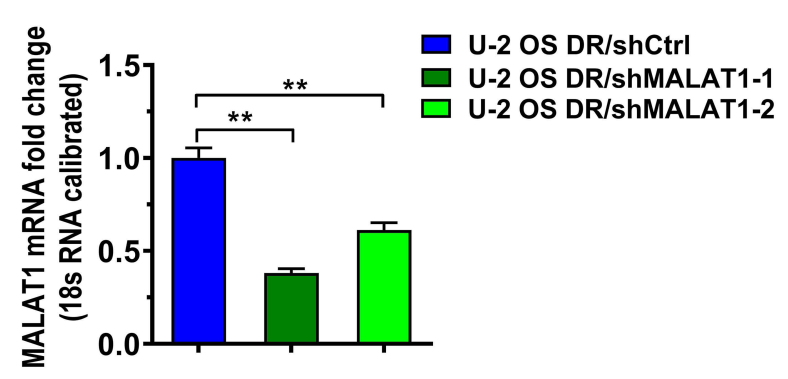

D
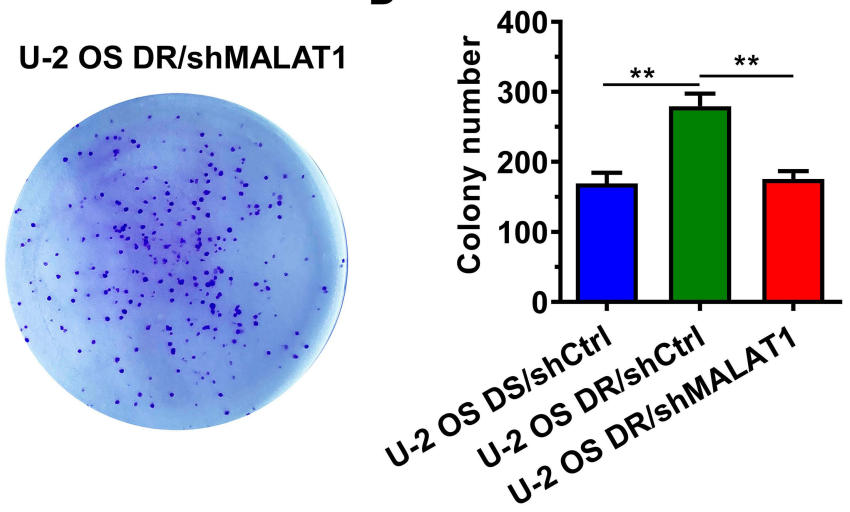

$\mathbf{F}$
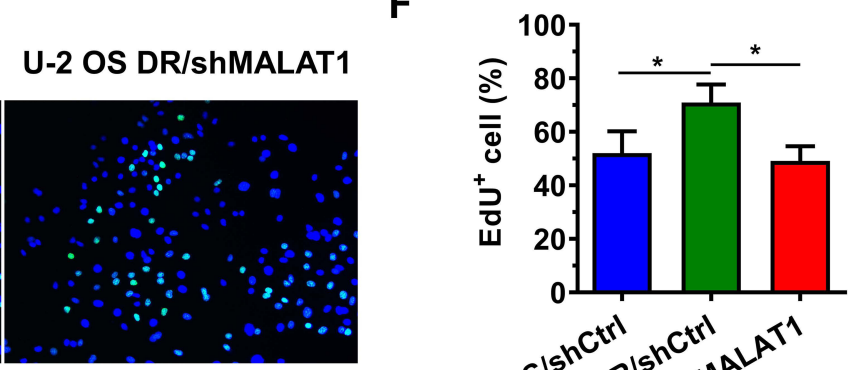

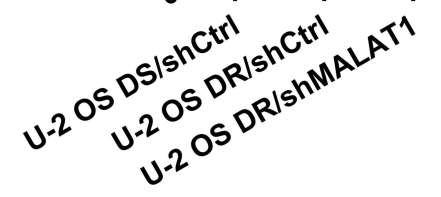

H

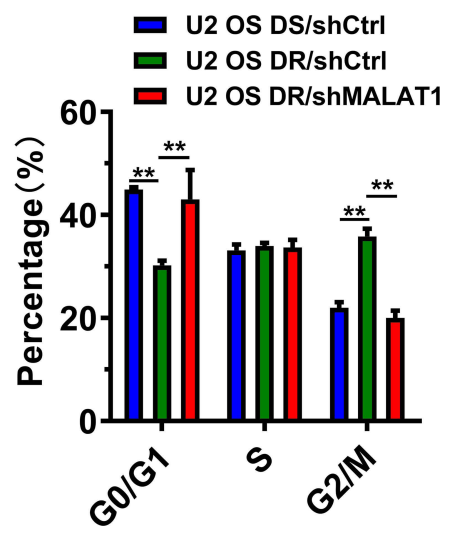

Figure 2 Changes in MALAT-I expression and the proliferation in U-2OS cells. (A) MALAT-I expression upregulated in doxorubicin-resistant U-2OS cells. (B) Target-I (U-2OS DR/shMALATI-I) demonstrated a higher efficiency than Target-2 (U-2OS DR/shMALATI-2) in downregulating MALAT-I expression in doxorubicin-resistant U-2OS cells. (C and D) The doxorubicin-resistant U-2OS osteosarcoma cells displayed more colonies than other cells. (E and $\mathbf{F}$ ) EdU assay showed that more EdU positive cells in doxorubicin-resistant cells. ( $\mathbf{G}$ and $\mathbf{H}$ ) The percentage of cells in the G2/M phase decreased and that in the GI phase increased while downregulating MALAT-I in the doxorubicin-resistant U-2OS cells.

Notes: ** $\mathrm{P}<0.01$. $* \mathrm{P}<0.05$

Abbreviations: DS, doxorubicin-sensitive U-2OS cells; DR, doxorubicin-resistant U-2OS cells; shCtrl, negative control; shMALATI, downregulating MALAT-I with shRNA. 


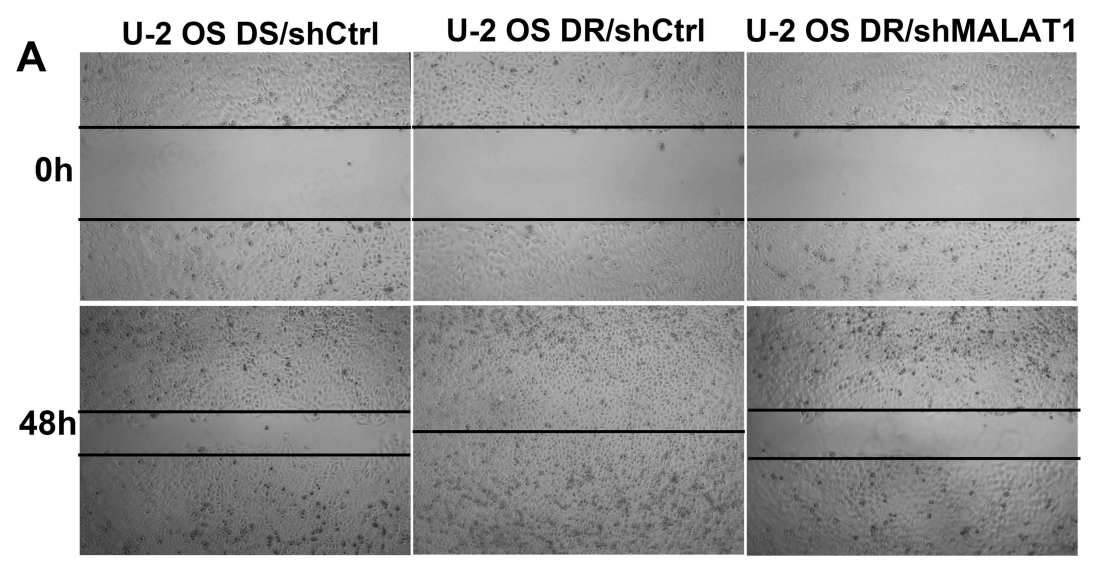

B
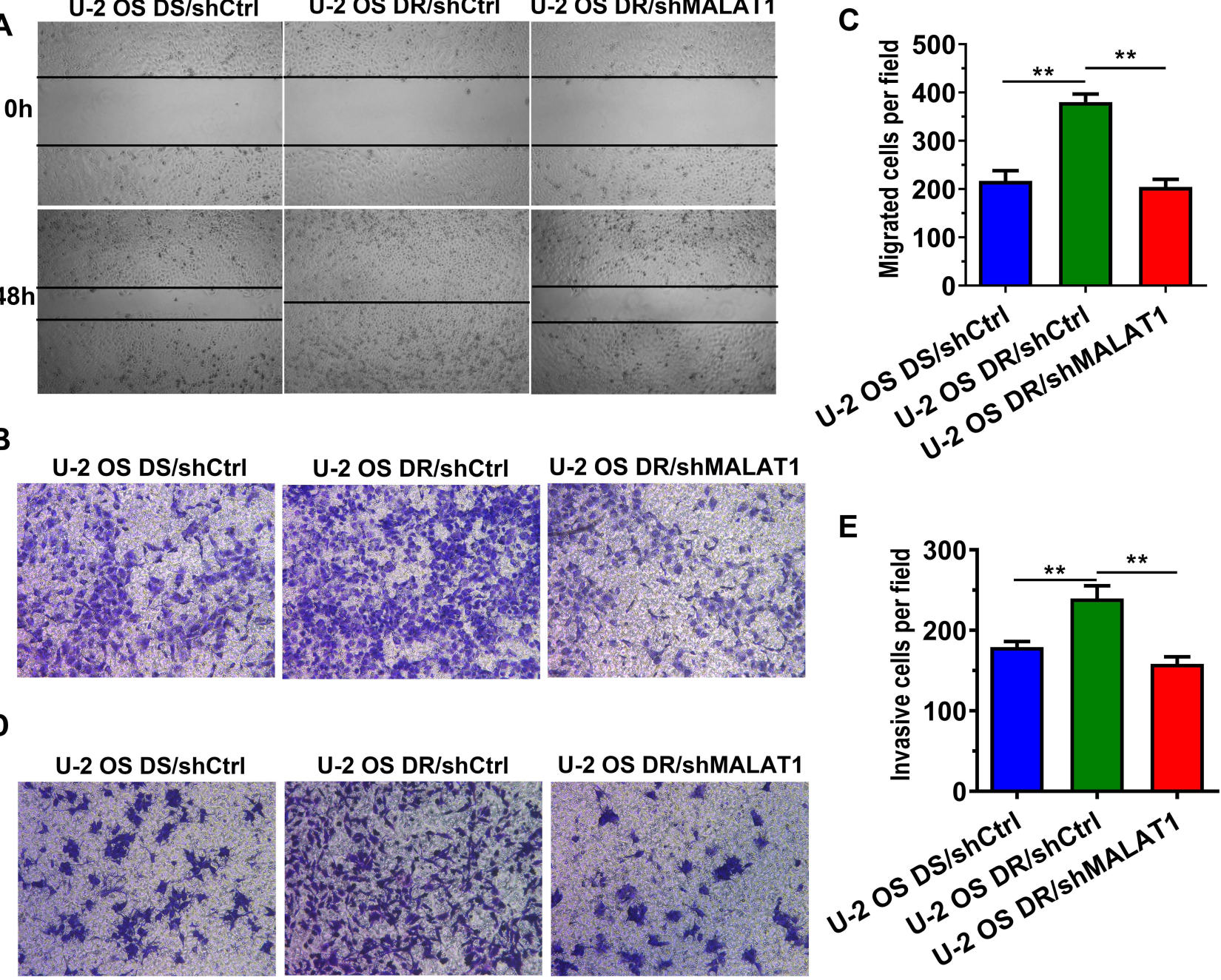

Figure 3 The doxorubicin-resistant cells exhibited better wound healing ability (A), migration ability (B and C), invasion ability (D and E), which could be reversed by downregulating MALAT-I.

Note: $* * \mathrm{P}<0.01$.

Abbreviations: DS, doxorubicin-sensitive U-2OS cells; DR, doxorubicin-resistant U-2OS cells; shCtrl, negative control; shMALATI, downregulating MALAT-I with shRNA.

reversed the expression of the related protein (Figure 4C and D).

\section{The Growth of Doxorubicin-Resistant Osteosarcoma Slowed Down with the Downregulating MALAT-I in vivo}

As shown in Figure 4E, the solid tumors in nude mice had different speeds of growth over time. The tumors of doxorubicin-resistant grew fastest, while the tumors of doxorubicin-sensitive and that of shMALAT1-doxorubicin-resistance had similar growth curves. All the tumors were collected on the 30th day (Figure 4F), the tumors of doxorubicinresistant had a higher average weight than the other two groups (Figure 4G).

\section{Discussion}

In the present study, we investigated the correlation between MALAT-1 expression and the chemotherapy resistance in the U-2OS osteosarcoma cells. Since perioperative chemotherapy for osteosarcoma is mainly the sequential use of chemotherapeutic drugs, including methotrexate, doxorubicin, cisplatin, and ifosfamide, we explored the influence of each chemotherapeutic drug on osteosarcoma cells at first. As a result, doxorubicin was the most likely drug associated with the upregulating MALAT-1 expression. In subsequent experiments, we identified significant upregulating MALAT-1 expression in the doxorubicin-resistant U-2OS osteosarcoma cells. The doxorubicin-resistant U-2OS cells displayed an 
A
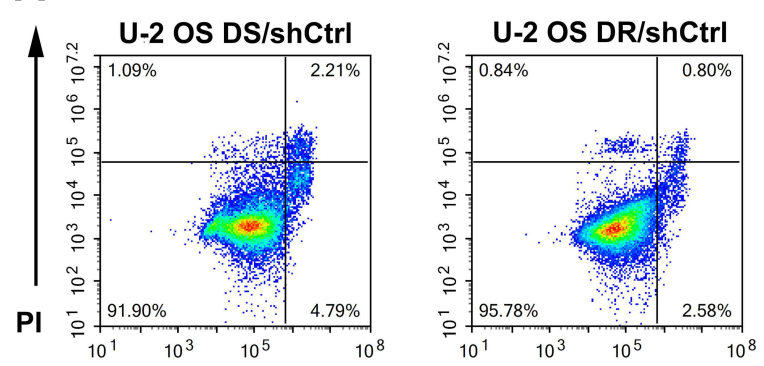

B
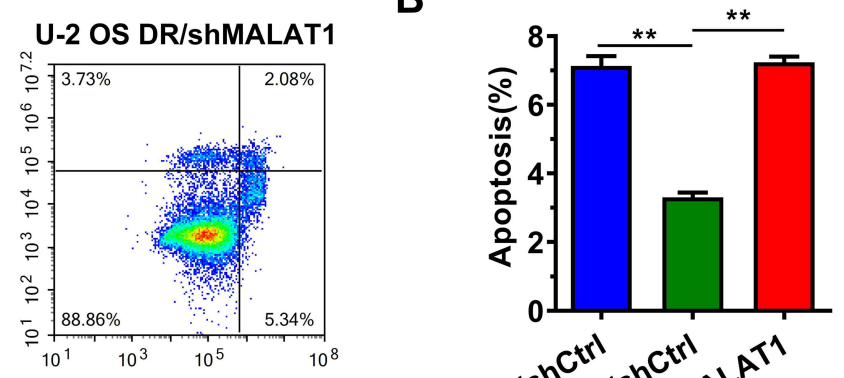

C
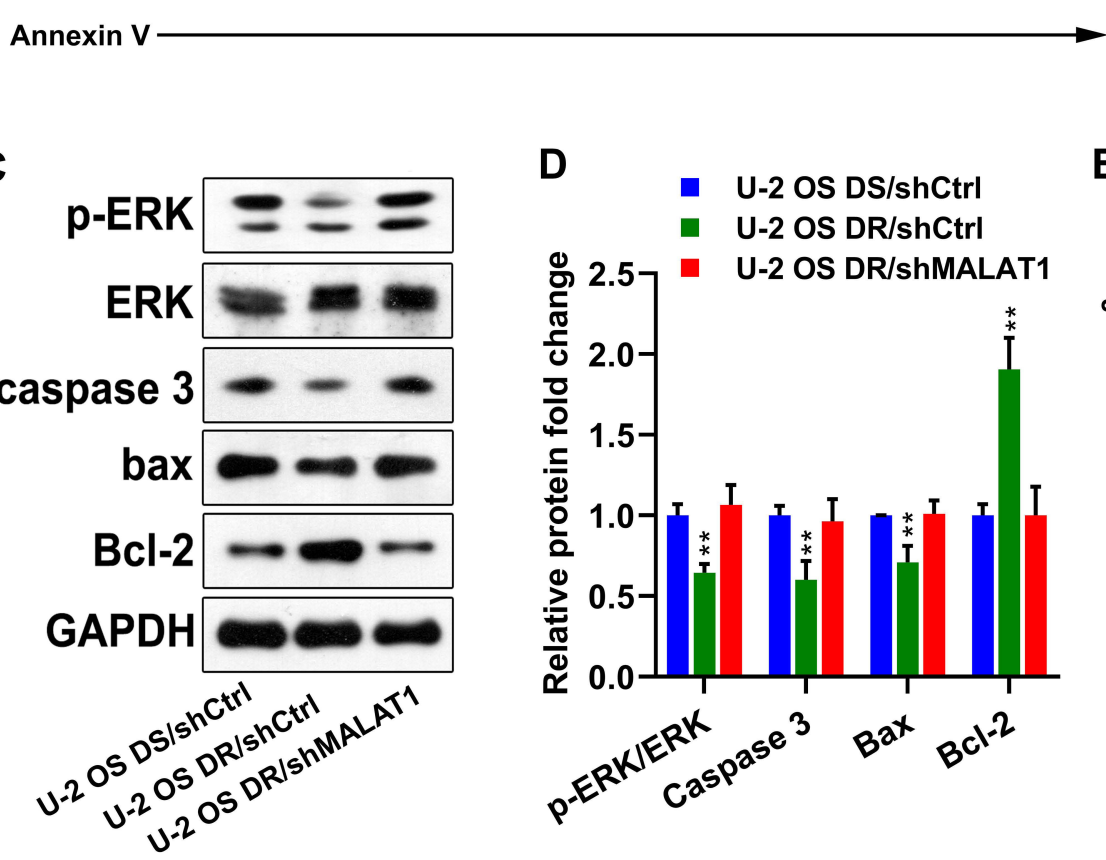

D

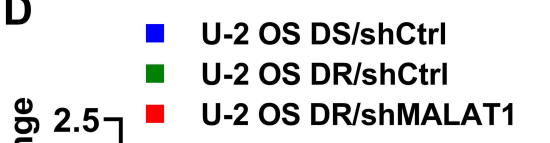

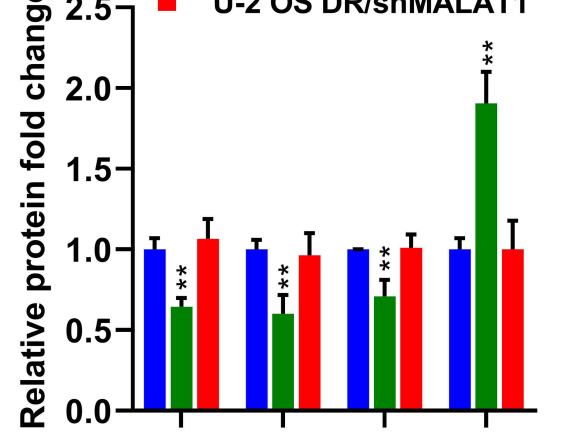

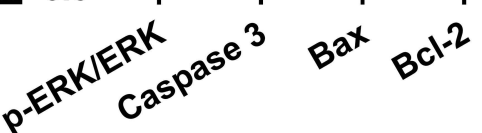

$\mathbf{E}$

E $\quad-4-2$ OS DS/shCtrl

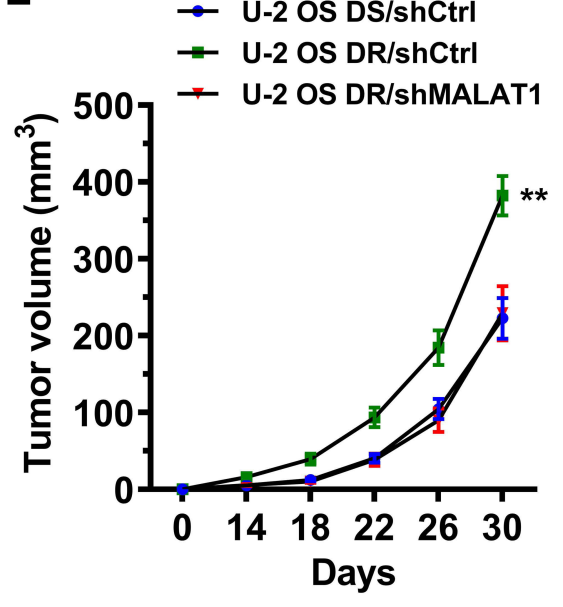

$\mathbf{F}$

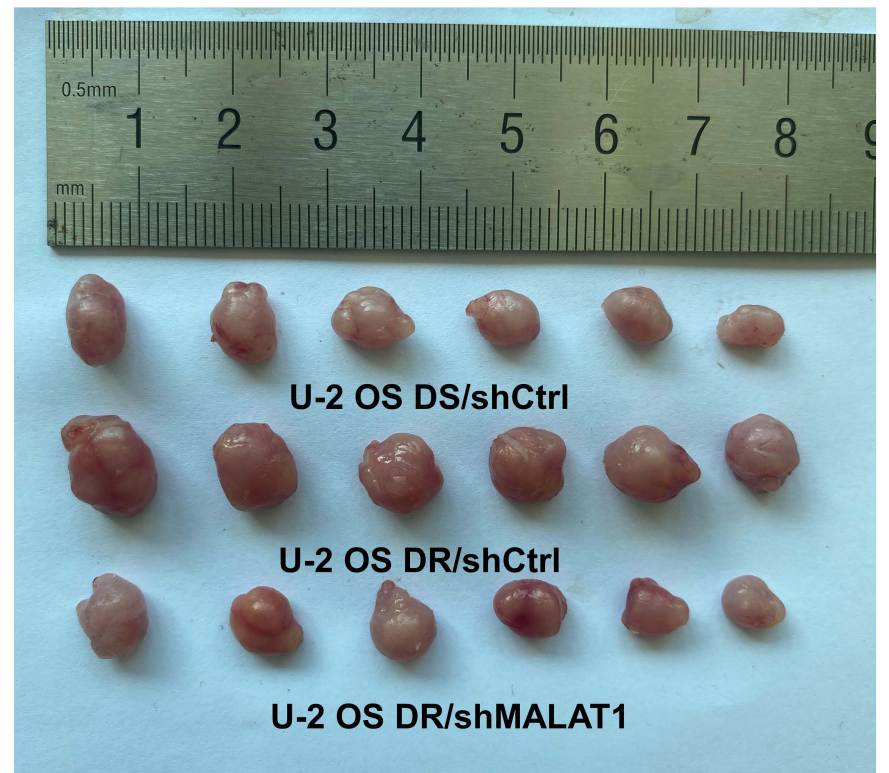

G

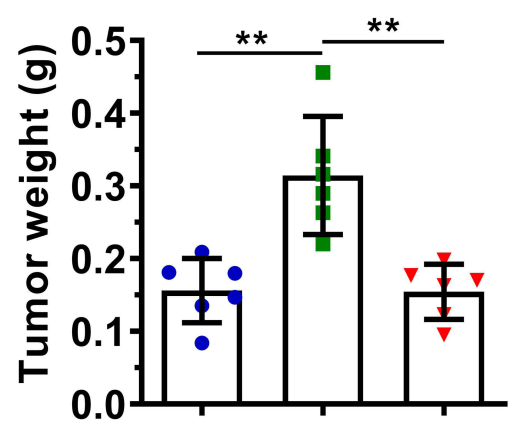

Figure 4 (A and B) The apoptosis in doxorubicin-resistant U-2OS cells was inhibited, which could be reversed by downregulating MALAT-I. (C and D) Western blot revealed that in the doxorubicin-resistant U-2OS cells, the pERK/ERK ratio, Caspase 3 expression, and Bax expression were decreased, while Bcl-2 expression was increased. (E) The growth curves of osteosarcoma cells in vivo. (F) The solid osteosarcoma was collected on the 30th day. (G) The average weight of doxorubicin-resistant solid osteosarcoma was higher than other tumors. Note: $* *$ P $<0.01$.

Abbreviations: DS, doxorubicin-sensitive U-2OS cells; DR, doxorubicin-resistant U-2OS cells; shCtrl, negative control; shMALATI, downregulating MALAT-I with shRNA; ERK, extracellular regulated protein kinases; Bcl-2, B-cell lymphoma-2; Bax, Bcl-2-associated X protein. 
increased proliferation, migration, and invasiveness ability, as well as decreased ERK phosphorylation and apoptosis, which could be reversed by downregulating MALAT-1 expression. Our study suggested that the chemotherapy resistance in osteosarcoma may be partly due to the upregulation of MALAT-1 and the reduction of ERK phosphorylation caused by chemotherapeutic drugs such as doxorubicin. The effects might result in tumor enhancements and inefficient chemotherapy response. As far as we know, this was the first research on the correlation between MALAT-1 and osteosarcoma chemotherapy resistance.

Recently, many researchers found that plenty of IncRNAs play an important role in cancer chemotherapy resistance, and MALAT-1 is one of the hot spots. The correlation between MALAT-1 and doxorubicin resistance has been demonstrated in several types of cancers, such as hepatocellular carcinoma ${ }^{15,19}$ and diffuse large B-cell lymphoma. ${ }^{16}$ Other lncRNAs that are related to doxorubicin resistance include lncRNA B4GALT1-AS1 in osteosarcoma, ${ }^{20}$ lncRNA Urothelial Carcinoma Associated 1(UCA1) in gastric cancer, ${ }^{21}$ linc-VLDLR in hepatocellular cancer, ${ }^{22}$ lncRNA growth arrest-specific 5 (GAS5) and HOTAIR (HOX transcript antisense RNA) in blanket transitional cell carcinoma, ${ }^{23,24}$ and lncRNABX537613 in breast cancer and liver cancer. ${ }^{25}$

Coincidentally, our study displayed that downregulating MALAT-1 can reverse the doxorubicin resistance in osteosarcoma. We further revealed that the ERK was involved in doxorubicin resistance and negatively correlated with MALAT-1 expression. Although previous literature has verified that the ERK phosphorylation can promote apoptosis and reduced the doxorubicin's efficacy in osteosarcoma, ${ }^{26-28}$ effect of MALAT-1 on the ERK/ MAPK pathway is not consistent. For example, some literature indicated that MALAT-1 activates ERK/MAPK pathway in hepatocellular carcinoma ${ }^{29}$ and cardiomyocytes, ${ }^{30}$ while another literature showed that MALAT-1 inhibits ERK/MAPK pathway in the nervous system. $^{31}$ Indeed, the potential mechanisms resulting in drug resistance are complex. Besides, the depth involved in current literature is also inconsistent. Multidisciplinary collaborative exploration, including but not limited to pharmacy, genomics, and biochemistry, is essential.

Notably, unlike Wang's results, which showed that MALAT-1 promotes cisplatin resistance in cervical cancer, ${ }^{14}$ no clear correlation between MALAT-1 and cisplatin resistance was observed in osteosarcoma. On one hand, it may owe to the differences in genetics and cytology between the two neoplasms. On the other hand, some details in the experiment which were not described may make a difference. Moreover, by studying the drug resistance mechanisms associated with MALAT-1 in the chemotherapeutic drugs of other cancers, inspiration may burst.

In addition to the above, the phenomenon exhibited in our study makes us think that some conclusions in previous literature may be one-sided. Previous researches illustrated that MALAT-1 is upregulated in osteosarcoma and is closely related to prognosis, cell invasion, and metastasis. ${ }^{9,10,12,32}$ Relevant mechanisms, such as E-calcium adhesive protein, ${ }^{32}$ micro-RNAs, ${ }^{9,10,12}$ and signaling pathways, ${ }^{11,13}$ were also explained. However, most of the literature did not describe when the specimens were taken (before or after chemotherapy) and which specimens met sampling requirements. Compared with other tumors, osteosarcoma has its characteristics. Firstly, most osteosarcoma patients have to receive neoadjuvant chemotherapy before surgery, which is different from some malignant tumors that are directly surgically removed after diagnosis. Specimens obtained in resection surgery may more likely display upregulation of MALAT-1. Secondly, as a tumor with high heterogeneity and rapid growth rate, there are usually necrotic areas inside the osteosarcoma. MALAT-1 expression may even be different in different regions of the same patient's tumor specimen. Furthermore, there is a possibility that the remaining live osteosarcoma cells after chemotherapy may come from the differentiation of residual cancer stem cells rather than drug-resistant cells, which call for clinical researches with sufficient patients and more in-depth basic researches.

Nevertheless, several limitations should be taken into account when interpreting our findings. Firstly, although the related mechanism was not fully explored, the experimental results partially explain the inherent reason for the poor prognosis with upregulated MALAT-1 and decreased ERK phosphorylation. Secondly, as the neoadjuvant chemotherapy for osteosarcoma is combination therapy, it is difficult to obtain tissue samples with definite doxorubicin resistance from patients.

In conclusion, our study displayed that doxorubicin may upregulate MALAT-1 in osteosarcoma. The upregulated MALAT-1 was found in the doxorubicin-resistant U-2OS osteosarcoma cells. Downregulating MALAT-1 expression in doxorubicin-resistant cells could reverse the proliferation, migration, invasiveness and promote apoptosis. ERK phosphorylation decreased in doxorubicin- 
resistant U-2OS osteosarcoma cells and could be reversed by downregulating MALAT-1. The upregulated MALAT-1 in osteosarcoma specimens may be the result of chemotherapy. Downregulating MALAT-1 may improve chemotherapy effects in osteosarcoma.

\section{Acknowledgments}

This work was supported by The 900th Hospital Project (No. 2018Q08, to Chang Liu), and "234 Subject Peak Climbing Plan" of The First Hospital Affiliated to the Naval Medical University (No. 2019YXK055, to Zhiwei Wang).

\section{Disclosure}

The authors report no conflicts of interest in this work.

\section{References}

1. Harrison DJ, Geller DS, Gill JD, Lewis VO, Gorlick R. Current and future therapeutic approaches for osteosarcoma. Expert Rev Anticancer. 2017;18(1):39-50. doi:10.1080/14737140.2018.1413939

2. Grinberg SZ, Posta A, Weber KL, Wilson RJ. Limb salvage and reconstruction options in osteosarcoma. Adv Exp Med Biol. 2020;1257:13-29. doi:10.1007/978-3-030-43032-0_2

3. Simpson E, Brown HL. Understanding osteosarcomas. JAAPA. 2018;31(8):15-19. doi:10.1097/01.JAA.0000541477.24116.8d

4. Wang J-Y, Yang Y, Ma Y, et al. Potential regulatory role of IncRNA-miRNA-mRNA axis in osteosarcoma. Biomed Pharmacother. 2020;121:109627. doi:10.1016/j.biopha.2019.109627

5. Harries LW. Long non-coding RNAs and human disease. Biochem Soc Trans. 2012;40(4):902-906. doi:10.1042/BST20120020

6. Kopp F. Molecular functions and biological roles of long non-coding RNAs in human physiology and disease. J Gene Med. 2019;21(8): e3104. doi:10.1002/jgm.3104

7. Zhang X, Hamblin MH, Yin KJ. The long noncoding RNA Malat1: its physiological and pathophysiological functions. RNA Biol. 2017;14(12):1705-1714. doi:10.1080/15476286.2017.1358347

8. Liu M, Yang P, Mao G, et al. Long non-coding RNA MALAT1 as a valuable biomarker for prognosis in osteosarcoma: a systematic review and meta-analysis. Int $J$ Surg. 2019;72:206-213. doi:10.1016/j.ijsu.2019.11.004

9. Sun Z, Zhang T, Chen B. Long non-coding RNA Metastasis-Associated Lung Adenocarcinoma Transcript 1 (MALAT1) promotes proliferation and metastasis of osteosarcoma cells by targeting c-Met and SOX4 via miR-34a/c-5p and miR-449a/ b. Med Sci Monit. 2019;25:1410-1422. doi:10.12659/MSM.912703

10. Duan G, Zhang C, Xu C, Xu C, Zhang L, Zhang Y. Knockdown of MALAT1 inhibits osteosarcoma progression via regulating the miR34a/cyclin D1 axis. Int $J$ Oncol. 2019;54(1):17-28. doi:10.3892/ijo.2018.4600

11. Cai X, Liu Y, Yang W, et al. Long noncoding RNA MALAT1 as a potential therapeutic target in osteosarcoma. J Orthop Res. 2016;34 (6):932-941. doi:10.1002/jor.23105

12. Fang D, Yang H, Lin J, et al. 17beta-estradiol regulates cell proliferation, colony formation, migration, invasion and promotes apoptosis by upregulating miR-9 and thus degrades MALAT-1 in osteosarcoma cell MG-63 in an estrogen receptor-independent manner. Biochem Biophys Res Commun. 2015;457(4):500-506. doi:10.1016/j.bbrc.2014.12.114
13. Chen Y, Huang W, Sun W, et al. LncRNA MALAT1 promotes cancer metastasis in osteosarcoma via activation of the PI3K-akt signaling pathway. Cell Physiol Biochem. 2018;51(3):1313-1326. doi:10.1159/ 000495550

14. Wang N, Hou MS, Zhan Y, Shen XB, Xue HY. MALAT1 promotes cisplatin resistance in cervical cancer by activating the PI3K/AKT pathway. Eur Rev Med Pharmacol Sci. 2018;22(22):7653-7659. doi:10.26355/eurrev_201811_16382

15. Yuan P, Cao W, Zang Q, Li G, Guo X, Fan J. The HIF-2alphaMALAT1-miR-216b axis regulates multi-drug resistance of hepatocellular carcinoma cells via modulating autophagy. Biochem Biophys Res Commun. 2016;478(3):1067-1073. doi:10.1016/j.bbrc.2016.08.065

16. Li LJ, Chai Y, Guo XJ, Chu SL, Zhang LS. The effects of the long non-coding RNA MALAT-1 regulated autophagy-related signaling pathway on chemotherapy resistance in diffuse large B-cell lymphoma. Biomed Pharmacother. 2017;89:939-948. doi:10.1016/j. biopha.2017.02.011

17. Li P, Zhang X, Wang H, et al. MALAT1 is associated with poor response to oxaliplatin-based chemotherapy in colorectal cancer patients and promotes chemoresistance through EZH2. Mol Cancer Ther. 2017;16(4):739-751. doi:10.1158/1535-7163.MCT-16-0591

18. Luo W, He H, Xiao W, et al. MALAT1 promotes osteosarcoma development by targeting TGFA via MIR376A. Oncotarget. 2016;7 (34):54733-54743. doi:10.18632/oncotarget.10752

19. Cao Y, Zhang F, Wang H, et al. LncRNA MALAT1 mediates doxorubicin resistance of hepatocellular carcinoma by regulating miR-3129-5p/Nova1 axis. Mol Cell Biochem. 2021;476(1):279-292. doi:10.1007/s11010-020-03904-6

20. Li Z, Wang Y, Hu R, Xu R, Xu W. LncRNA B4GALT1-AS1 recruits HuR to promote osteosarcoma cells stemness and migration via enhancing YAP transcriptional activity. Cell Prolif. 2018;51(6): e12504. doi:10.1111/cpr.12504

21. Fang Q, Chen X, Zhi X. Long non-coding RNA (LncRNA) Urothelial Carcinoma Associated 1 (UCA1) increases multi-drug resistance of gastric cancer via downregulating miR-27b. Med Sci Monit. 2016;22:3506-3513. doi:10.12659/MSM.900688

22. Takahashi K, Yan IK, Wood J, Haga H, Patel T. Involvement of extracellular vesicle long noncoding RNA (linc-VLDLR) in tumor cell responses to chemotherapy. Mol Cancer Res. 2014;12 (10):1377-1387. doi:10.1158/1541-7786.MCR-13-0636

23. Zhang H, Guo Y, Song Y, Shang C. Long noncoding RNA GAS5 inhibits malignant proliferation and chemotherapy resistance to doxorubicin in bladder transitional cell carcinoma. Cancer Chemother Pharmacol. 2017;79(1):49-55. doi:10.1007/s00280016-3194-4

24. Shang C, Guo Y, Zhang H, Xue YX. Long noncoding RNA HOTAIR is a prognostic biomarker and inhibits chemosensitivity to doxorubicin in bladder transitional cell carcinoma. Cancer Chemother Pharmacol. 2016;77(3):507-513. doi:10.1007/s00280-016-2964-3

25. Jiang M, Huang $\mathrm{O}, \mathrm{Xie} Z$, et al. A novel long non-coding RNA-ARA: adriamycin resistance-associated. Biochem Pharmacol. 2014;87 (2):254-283. doi:10.1016/j.bcp.2013.10.020

26. Ma L, Xu Y, Xu X. Targeted MEK inhibition by cobimetinib enhances doxorubicin's efficacy in osteosarcoma models. Biochem Biophys Res Commun. 2020;529(3):622-628. doi:10.1016/j.bbrc.2020.06.082

27. Noh K, Kim KO, Patel NR, et al. Targeting inflammatory kinase as an adjuvant treatment for osteosarcomas. J Bone Joint Surg Am. 2011;93(8):723-732. doi:10.2106/JBJS.J.00302

28. Zheng L, Fang S, Hui J, et al. Triptonide modulates MAPK signaling pathways and exerts anticancer effects via ER stress-mediated apoptosis induction in human osteosarcoma cells. Cancer Manag Res. 2020;12:5919-5929. doi:10.2147/CMAR.S258203

29. Xie SJ, Diao LT, Cai N, et al. mascRNA and its parent lncRNA MALAT1 promote proliferation and metastasis of hepatocellular carcinoma cells by activating ERK/MAPK signaling pathway. Cell Death Discov. 2021;7(1):110. doi:10.1038/s41420-021-00497-x 
30. Fan YZ, Huang H, Wang S, Tan GJ, Zhang QZ. Effect of lncRNA MALAT1 on rats with myocardial infarction through regulating ERK/ MAPK signaling pathway. Eur Rev Med Pharmacol Sci. 2019;23 (20):9041-9049. doi:10.26355/eurrev_201910_19306

31. Shi YL, Wang Q, Wei JC. Influence of IncRNA-MALAT1 on neuronal apoptosis in rats with cerebral infarction through regulating the ERK/MAPK signaling pathway. Eur Rev Med Pharmacol Sci. 2019;23(18):8039-8048. doi:10.26355/eurrev_201909_19020
32. Huo Y, Li Q, Wang X, et al. MALAT1 predicts poor survival in osteosarcoma patients and promotes cell metastasis through associating with EZH2. Oncotarget. 2017;8(29):46993-47006. doi:10.18632/ oncotarget. 16551

\section{Publish your work in this journal}

Cancer Management and Research is an international, peer-reviewed open access journal focusing on cancer research and the optimal use of preventative and integrated treatment interventions to achieve improved outcomes, enhanced survival and quality of life for the cancer patient.
The manuscript management system is completely online and includes a very quick and fair peer-review system, which is all easy to use. Visit http://www.dovepress.com/testimonials.php to read real quotes from published authors. 